\title{
Denoised Modified Incoherent Signal Subspace Method for DOA of Coherent Signals
}

\author{
Bin $\mathrm{Hu}$, Xue Jun Li, Peter Han Joo Chong \\ Department of Electrical and Electronic Engineering \\ Auckland University of Technology \\ Auckland, New Zealand \\ \{bin.hu, xuejun.li, peter.chong\}@aut.ac.nz
}

\begin{abstract}
Incoherent signal subspace method (ISM) is ineffective to deal with direction of arrival (DOA) of coherent wideband signals. Although modified methods were proposed to solve this drawback, such as ISM based on modified MUSIC (MMUSIC-ISM), its performance will seriously deteriorate when the signal-to-noise ratio (SNR) is low. This paper proposes a method to construct the covariance matrix, namely denoised modified ISM (DMISM), which attempts to effectively reduce the influence of stationary white Gaussian noise. Numerical experiments affirm that DMISM outperforms MMUSIC-ISM.
\end{abstract}

Keywords-modified ISM, coherent signals, low SNR, spectrum peaks, denoised matrix

\section{INTRODUCTION}

Incoherent Signal Subspace Method (ISM) [1] is a conventional method for estimating direction of arrival (DOA) estimation of wideband signals. According to ISM, a wideband signal can be considered as the superposition of numerous narrowband signals at different frequencies [2]. Therefore, algorithms dealt with DOA in narrowband coherent signals such as Modified Multiple Signal Classification (MMUSIC) [3] can be applied to each separated narrowband component, then DOA can be obtained by averaging the estimation result of each component. However, the performance of DOA estimation algorithms is largely affected by the signal-to-noise ratio (SNR).

In this paper, we propose denoised modified ISM (DMISM) to minimize the influence of additive stationary white Gaussian noise mingled with incident signals. We replace initial covariance matrix in each narrowband component with the denoised one to achieve performance improvement in DOA estimation. Estimation results of DMISM can be detected easily due to its salient spectrum peaks in DOA spatial spectrumNumerical experiments affirm that the proposed DMISM outperforms existing MMUSIC-ISM methods, especially for low SNR scenarios.

\section{Signal Model}

Assuming that $K$ wideband signals, generated by a linear frequency modulation (LFM) [4] function, whose frequency ranging from $f_{L}$ to $f_{H}$, embedded in stationary white Gaussian noise impinging on an uniform linear array (ULA) consists of $N$ omnidirectional sensors from multiple bearings $\theta_{i}, i=1, \cdots, K$.
Wideband signal can be considered as superposition of $J$ narrowband signals

$$
X\left(f_{j}\right)=A\left(f_{j}, \theta\right) S\left(f_{j}\right)+N\left(f_{j}\right)
$$

where $A\left(f_{j}, \theta\right)=\left[a\left(f_{j}, \theta_{1}\right), a\left(f_{j}, \theta_{2}\right), \ldots, a\left(f_{j}, \theta_{K}\right)\right], a\left(f_{j}, \theta_{i}\right)=\left[e^{-j 2 \pi f_{j} \tau_{1} \theta_{i}}\right.$, $\left.e^{-j 2 \pi f_{j} \tau_{2} \theta_{i}}, \ldots, \quad e^{-j 2 \pi f_{j} \tau_{N} \theta_{i}}\right], \quad S\left(f_{j}\right)=\left[S_{1}\left(f_{j}\right), \quad S_{2}\left(f_{j}\right), \ldots, \quad S_{K}\left(f_{j}\right)\right]^{T}$, $N\left(f_{j}\right)=\left[N_{1}\left(f_{j}\right), N_{2}\left(f_{j}\right), \ldots, N_{N}\left(f_{j}\right)\right]^{T}$. The covariance matrix [5] of received signal vector in each narrowband frequency bin is defined by

$$
R\left(f_{j}\right)=A\left(f_{j}, \theta\right) R_{S} A^{H}\left(f_{j}, \theta\right)+\sigma_{n}^{2} I
$$

MMUSIC-ISM performs DOA estimation of coherent wideband signals by first applying MMUSIC [6] in $R\left(f_{i}\right)$ to obtain DOA estimation in each frequency bin, and then taking an average of the $J$ DOA results.

\section{DENOISED MODIFIED ISM}

By considering two coherent signals impinging on ULA consisting of $N$ sensors, the eigenvalue decomposition of reconstructed Toeplitz matrix [7] [8] $R_{T}$ is given by

$$
R_{T}=U \Lambda U^{H}
$$

where $U=\left[U_{1}, U_{2}\right], \Lambda=\left[\begin{array}{ll}\Lambda_{1} & \\ & \Lambda_{2}\end{array}\right] \cdot \Lambda_{1}=\operatorname{diag}\left(\lambda_{1}, \lambda_{2}\right)$ are two major eigenvalues, their corresponding eigenvectors are $U_{1}=\left[u_{1}, u_{2}\right] . \Lambda_{2}=\operatorname{diag}\left(\lambda_{3}, \lambda_{4}, \cdots, \lambda_{N}\right)$ are the remaining eigenvalues, their corresponding eigenvectors are $U_{2}=\left[u_{3}, u_{4}, \cdots, u_{N}\right]$. By denoising, a matrix $R_{S}^{\prime}$ can be obtained as

$$
R_{S}^{\prime}=U_{1} \cdot\left(\Lambda_{1}-\bar{\lambda} \cdot I\right) \cdot U_{1}^{H}
$$

where $\bar{\lambda}=\frac{1}{N-2} \sum_{i=3}^{N} \lambda_{i}$. Note that the reconstructed matrix $R_{S}^{\prime}$ might not be a Toeplitz matrix. We need to reconstruct $R_{S}^{\prime}$ into a Toeplitz matrix $T$ by

$$
\min \left\|T-R_{S}^{\prime}\right\|_{F}, \text { s.t. } \frac{1}{N-2} \sum_{i=3}^{N}\left|\lambda_{i}\right|<\hat{\sigma}_{n}^{2}
$$


where $\hat{\sigma}_{n}^{2}$ denotes the arbitrary small noise threshold.

The optimal approximation matrix $T$ is obtained by performing the aforementioned procedures on $\hat{R}$ and $R_{S}^{\prime}$. As compared to $\hat{R}, T$ has several advantages, such as full rank and minimized influence of additive noise. Consequently, $T$ leads to salient peak values in the DOA spatial spectrum. We can obtain the DOA estimation through substituting $T$ for $\hat{R}$, and search for spectrum peaks using singular value decomposition.

\section{Simulation Results}

In our numerical experiments, we adopt a ULA consisting of $N$ sensors. The spectrum peak searching ranging from $-90^{\circ}$ to $90^{\circ}$ is used with a step size of $0.1^{\circ}$. The number of snapshots is $N_{S}$. We first compare the proposed DMISM with MMUSIC-ISM and ISM in terms of peak detection. The DOAs of two coherent incident signals are $\left[5^{\circ} 14^{\circ}\right]$, whose frequency ranging from $8000 \mathrm{~Hz}$ to $12000 \mathrm{~Hz}, N=10, J=50$, the arbitrary small noise threshold $\hat{\sigma}_{n}^{2}=10^{-6}$, the number of snapshots $N_{S}=200$. Two considered SNR values are $10 \mathrm{~dB}$ and $-5 \mathrm{~dB}$.

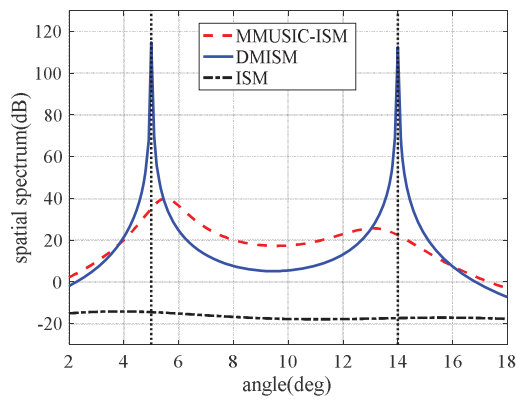

Fig. 1 Comparison among ISM, MMUSIC-ISM, DMISM with SNR=10dB

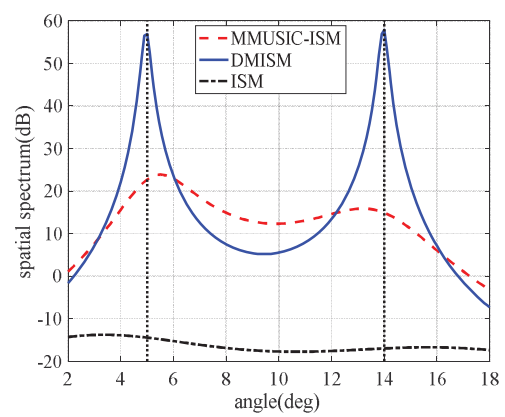

Fig. 2 Comparison among ISM, MMUSIC-ISM, DMISM with SNR= $-5 \mathrm{~dB}$

Fig. 1 and Fig. 2 compare the performance of DMISM, MMUSIC-ISM and ISM under different SNR levels. Obviously, ISM failed to estimate DOA for coherent signals. As it can be seen from Fig. 1, DMISM and MMUSIC-ISM are effective when $\mathrm{SNR}=10 \mathrm{~dB}$. However, when $\mathrm{SNR}$ is reduced to $-5 \mathrm{~dB}$, MMUSIC-ISM fail to detect the peaks. On the contrary, the spectrum peaks of DMISM are easily discerned. Despite of the slight deviation of DOA estimation for the $\mathrm{SNR}=-5 \mathrm{~dB}$ scenario, DMISM achieves good DOA estimations.

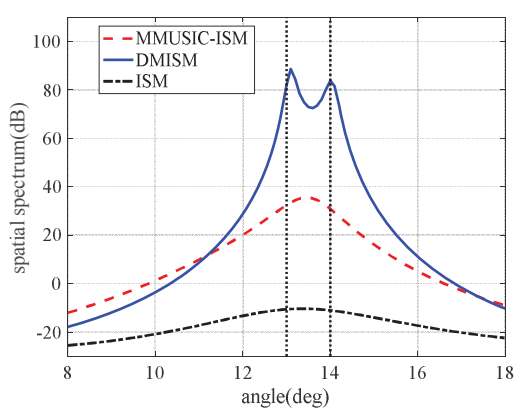

Fig. 3. Performance comparison among ISM, MMUSIC-ISM, DMISM with spatially close signals DOAs $\left[13^{\circ} 14^{\circ}\right]$

Fig. 3 shows experimental results of these three algorithms with two close incident signals. Two spatially coherent signals from DOAs $\left[13^{\circ} 14^{\circ}\right]$ impinging on a ULA with $N=16$ array sensors, $\mathrm{SNR}=10 \mathrm{~dB}$. The number of snapshots $N_{S}=200$. MMUSIC-ISM failed to observe the direction angles of two close incident signals, and ISM even could not identify the direction angles. However, the proposed DMISM still achieves two spectrum peaks and estimated DOA values are adequately close to the source values.

\section{CONCLUSION}

We proposed denoised modified ISM by exploiting optimal approximation. Numerical experiment results demonstrated that the proposed DMISM outperforms MMUSIC-ISM in terms of spectrum peaks, even when two spatial incident signals are close. Furthermore, when the SNR is low, the superiority of the proposed DMISM over MMUSIC-ISM becomes more prominent.

\section{REFERENCE}

[1] G. Su and M. Morf, "The signal subspace approach for multiple wideband emitter location," IEEE Transactions on Acoustics, Speech, and Signal Processing, vol. 31, pp. 1502-1522, 1983.

[2] J. Zhang, J. Dai, and Z. Ye, "An extended TOPS algorithm based on incoherent signal subspace method," Signal Processing, vol. 90, pp. 3317-3324, 2010.

[3] F. Guo, H. Liu, J. Huang, X. Zhang, X. Zu, B. Li, et al., "Design of a direction-of-arrival estimation method used for an automatic bearing tracking system," Sensors, vol. 16, pp. 1145, 2016.

[4] K. Cui, W. Wu, J. Huang, X. Chen, and N. Yuan, "DOA estimation of LFM signals based on STFT and multiple invariance ESPRIT," AEU Int. J. Electron. Commun., vol. 77, pp. 10-17, 2017.

[5] L. Wang, L. Zhao, G. Bi, C. Wan, L. Zhang, and H. Zhang, "Novel wideband DOA estimation based on sparse Bayesian learning with Dirichlet process priors," IEEE Transactions on Signal Processing, vol. 64, pp. 275-289, 2016.

[6] H. Paaso, A. Mämmelä, D. Patron, and K. R. Dandekar, "Modified MUSIC algorithm for DoA estimation using CRLH leaky-wave antennas," in Proc of CROWNCOM'13, 2013, pp. 166-171.

[7] J. Bai, X. Shen, H. Wang, and Y. Liu, "Improved toeplitz algorithms to coherent sources DOA estimation," in Proc of ICMTMA, Changsha City, China, 2010, pp. 442-445.

[8] X. Fan, C. Zhou, Y. Gu, and Z. Shi, "Toeplitz matrix reconstruction of interpolated coprime virtual array for DOA estimation," in Vehicular Technology Conference (VTC Spring), 2017 IEEE 85th, 2017, pp. 1-5. 